\title{
Research on inertial response control technology of high voltage direct hanging energy storage system
}

\author{
Huaidong Yan ${ }^{1}$, Haoyuan $\mathrm{Li}^{2 *}$, Lihua $\mathrm{Cai}^{1}$, Anping $\mathrm{Hu}^{2}$, Zhao $\mathrm{Liu}^{3}$ \\ ${ }^{1}$ State Grid Yancheng Power Supply Company, Yancheng, Jiangsu, 224000, China \\ ${ }^{2}$ China Electric Power Research Institute Co., Ltd, Nanjing, Jiangsu, 210003, China \\ ${ }^{3}$ Nanjing University of Science and Technology, Nanjing, Jiangsu, 210014, China
}

\begin{abstract}
The high voltage direct hanging energy storage system can effectively solve the problems of fluctuation and intermittence caused by environmental factors, and improve the ability of power system to absorb new energy. By controlling the energy storage, the new energy station has certain inertia and damping characteristics, so that the new energy power station can be connected to the grid friendlier. Starting from the time scale division method of inertial response control, this paper studies the energy demand of inertial response of large-scale new energy power station in different time scales, and gives the inertial response control strategy under different time scales. The model of high voltage direct hanging energy storage system is established, and the inertia response characteristics control technology is verified.
\end{abstract}

\section{Introduction}

Energy is an important factor to maintain human survival and development. The increasing population and the development of social economy need a lot of energy as the driving force. It not only brings great convenience and benefits to human beings, but also brings many problems, such as energy shortage and fossil energy pollution. The development and utilization of renewable energy is of great significance to ensure energy security and solve environmental problems [1]. With the rapid development of new energy such as photovoltaic and wind power, the proportion of new energy is gradually increasing, and the penetration rate is increasing day by day, while the installed proportion of traditional synchronous generator (SG) represented by thermal power is gradually reduced, and the energy structure tends to be diversified. The new energy generation systems represented by photovoltaic and wind power are mostly power electronic interfaces and connected to the grid in the mode of maximum power point tracking (MPPT). The process of grid connection almost contains no rotary inertia [2]. Therefore, the rotary inertia and system damping of the power system are relatively reduced, and they are more vulnerable to voltage fluctuations, power fluctuations and system failures.

The new energy is not friendly to the power grid, mainly because the power cannot be adjusted under the MPPT operation mode, and the control method of power electronic devices connected to the power grid does not have the inertia of synchronous generator. Therefore, it is considered to improve the energy storage technology and apply it to the new energy grid connected device, so as to reduce the power fluctuation of the power generation system and have the ability of frequency modulation [3]. It can effectively solve the problems of fluctuation and intermittence of new energy power generation caused by environmental factors, and improve the ability of power system to absorb new energy. By simulating the external characteristics of synchronous generator, the new energy station has the ability of active power frequency regulation and reactive power voltage regulation, which can effectively enhance the frequency stability and voltage stability of power system, and realize safe, friendly, stable and reliable grid connection [4].

In order to solve the above problems, the performance and technology of the traditional grid connected inverter should be greatly improved. Some advanced control strategies for the inverter interface need further research and development to improve the inertia response and damping characteristics of the grid connected new energy power station, and escort the safe and stable operation of the large power grid. Therefore, this paper focuses on the grid connection technology of new energy power station based on high voltage direct hanging energy storage system [5], and puts forward the key technology of inertial response control for energy storage to enhance the active support capacity of new energy power station, so that the new energy power station can actively support the stability of grid voltage and frequency, and promote the development of friendly access technology of new energy sources. 


\section{Inertial response characteristics of high voltage direct hanging energy storage system}

\subsection{Model of high voltage direct hanging energy storage station}

The station level active power control model belongs to the upper level control of wind farm, and the output is station level active power control command. This model provides two control modes: open-loop control and closed-loop PI control, which can be used for simulation analysis of primary frequency regulation of wind farm. The model block diagram is shown in Fig. 1.

The station level reactive power control model also belongs to the upper level control of wind farm, and the output is station level reactive power control command. The model provides constant voltage control, reactive power droop control, constant power factor control and constant reactive power control. It can be used for simulation analysis of station level voltage regulation of wind farm. The model block diagram is shown in Fig. 2.

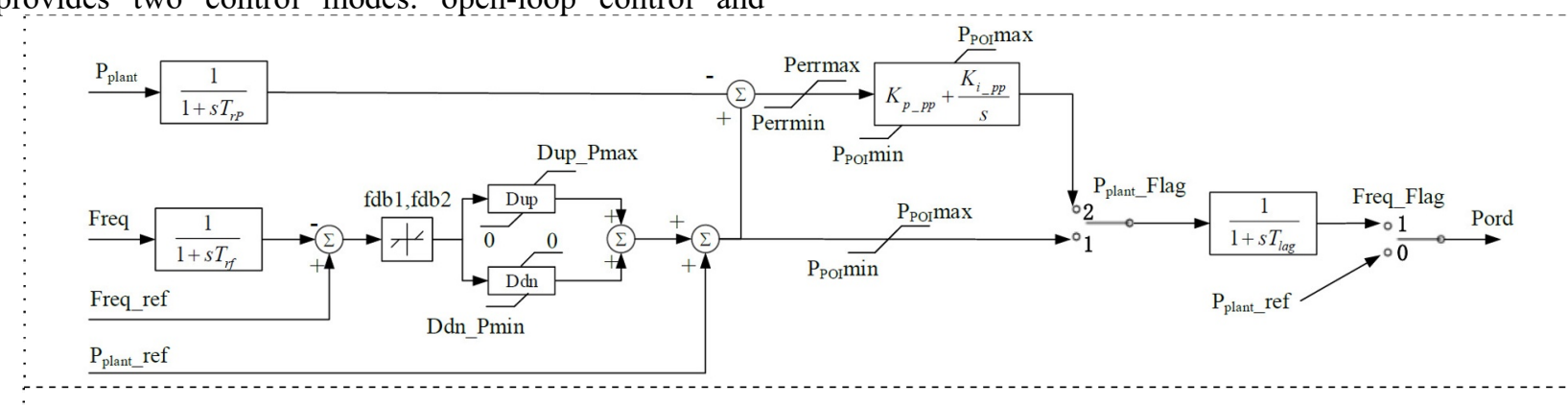

Figure 1. Station level active power control mode.

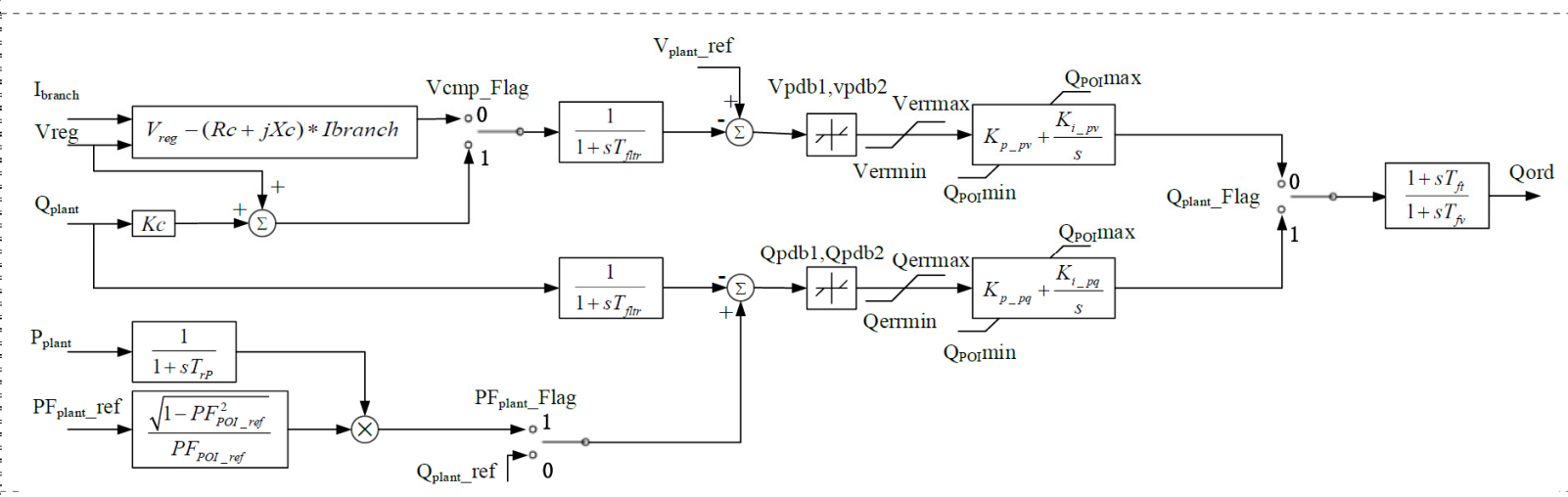

Figure 2. Station level reactive power control model.

The active power control model of converter level belongs to local level control of wind turbine, and the input is the active power command $\left(P_{\text {ord_trq }}\right)$ given by torque control model. The model provides two control modes, i.e., open-loop control and closed-loop PI control. The model output is the active current control command $\left(I_{\text {pcmd }}\right)$, and the block diagram is shown in Fig. 3.

The converter level reactive power control model belongs to the local level control of wind turbine, and the reactive power control commands can be as follows: 1 ) the reactive power command calculated by the constant power factor of the fan; 2) the initial value of the reactive power of the fan; 3 ) the reactive power command given by the station level control. The model provides open-loop control, reactive power and voltage coordinated control, reactive power PI control and fixed initial reactive current control. In addition, the model also provides additional injection current control. The model output is the reactive current control command $\left.\left(\mathrm{I}_{q \mathrm{cmd}}\right)^{\prime}\right)$, and the block diagram is shown in Fig. 4. 


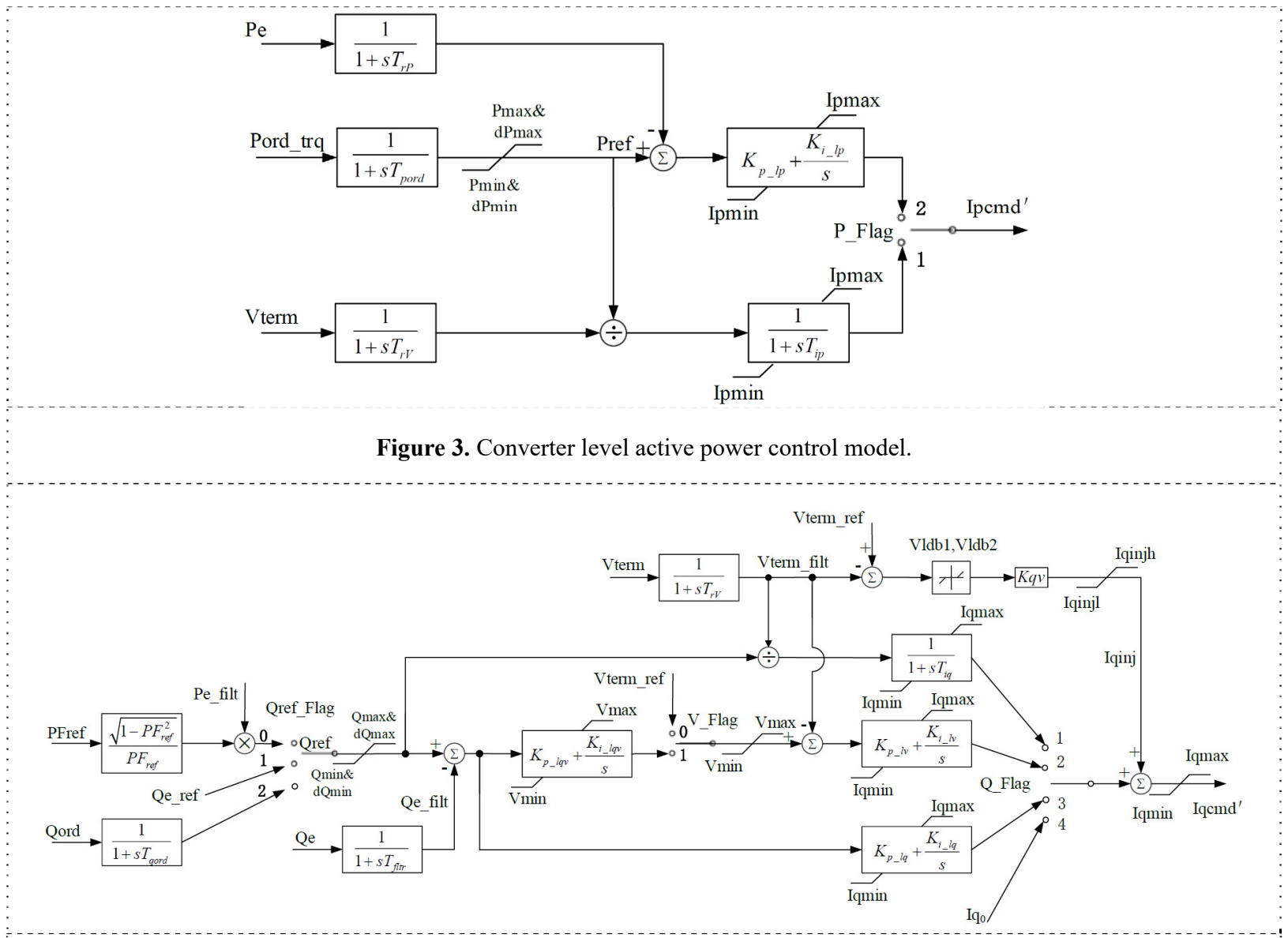

Figure 4. Converter level reactive power control model.

\subsection{Inertial response time scale characteristics of frequency response}

The power system stability is caused by external disturbance of power system. The relationship between power system inertia and inertia is as follows: the effect of inertia is embodied in inertia, the energy source of inertia is inertia, and the size of inertia can be measured by inertia.

When the power system has fault disturbance, power impact and frequency fluctuation, it depends on the rotational inertia of a large number of rotating equipment, which is called moment of inertia. The moment of inertia represents the dynamic stability of the system. For the new energy power generation without virtual inertia control function, the equivalent moment of inertia of wind turbine blade is very small, photovoltaic and energy storage are no rotating static components. The low inertia problem of power grid under high permeability will be more severe than that of wind power. The reasonable definition of virtual inertia of new energy station needs to expand the energy source of inertia, use the rapid charge and discharge of energy storage device to adjust the active power, suppress the frequency mutation, and obtain the inertial response to the system frequency change.

In the primary frequency regulation time scale, the inertia response of high voltage direct hanging energy storage system aims to simulate the external frequency and voltage characteristics of traditional synchronous generator when it is adjusted by its own moment of inertia and governor. These disturbance factors include power fluctuation of new energy power station, abnormal voltage and frequency flicker caused by transient short circuit of high voltage direct hanging energy storage system. The response time and regulation time of primary frequency regulation of large-scale power station based on energy storage is generally faster than that of traditional generator set. Especially, the response time of electrochemical energy storage system based on power electronic converter is millisecond level, and the regulation time is basically at second level. Therefore, the time scale of primary frequency regulation is shorter than that of conventional generator set.

On the time scale of secondary frequency regulation, the power system can shorten the time and response accuracy of secondary frequency regulation by using the characteristics of battery and other energy storage equipment. The difference between the inertia response of high voltage direct hanging energy storage system on the time scale of secondary frequency regulation and that on the time scale of primary frequency regulation is that in the time scale of primary frequency regulation, energy storage can restrain the rapid change of system frequency, so as to increase the equivalent moment of inertia; on the time scale of secondary frequency regulation, energy storage plays a role in rapidly coordinating active power output to achieve maintenance system frequency to rated 
value purpose.

\subsection{Analysis of inertial response energy demand of high voltage direct hanging energy storage in different time scales}

In the traditional research of converter grid connection, the power grid is usually modeled as a single machine infinite bus system, whose frequency and voltage are constant and not affected by the outside world. Considering that the inertia response of new energy power plants is generally required when they are connected to the weak inertia power grid, and the characteristics of weak inertia power grid is that the frequency of power grid will change when the load power fluctuates in the network. When the inertia of the power grid is very weak and the load power fluctuation is large, the frequency change rate of the power grid may even exceed the tolerance of generators and some sensitive loads Limit. Therefore, the inertia model of power station should be able to reflect the inertia characteristics of the new power grid.

The frequency response model of power grid shown in (1) is proposed to represent the non-ideal power grid with inertia and damping characteristics

$$
2 H \frac{\mathrm{d} \omega}{\mathrm{d} t}=P-P_{g}=P-\left[P_{g 0}+\Delta P_{g}-K_{g}\left(\omega_{N}-\omega\right)\right]
$$

where $H$ represents the inertia effect of the system, $P_{g}$ represents the total power consumed in the power grid including load, disturbance and frequency response characteristics, $K_{g}$ describes the frequency response characteristics of the power grid, $P$ represents the power transmitted by the generation unit to the grid, $\Delta P_{g}$ describes the disturbance characteristics of the power grid.

Inertial response, which is to suppress the frequency change as the goal, according to the frequency change rate of the power grid to adjust its own output power. Referring to the rotor inertia response characteristics of SG, the new energy can be controlled to have similar inertial response characteristics, i.e., the power change is directly proportional to the frequency change rate. Therefore, under the grid side power disturbance of $\Delta P_{g}$, the inertia support power $P_{\mathrm{FR}}$ provided is as (2), which is the basic characteristic of inertial response provided by new energy power station.

$$
P_{\mathrm{FR}}=-K_{\mathrm{d}} \frac{\mathrm{d} \omega}{\mathrm{d} t}
$$

When the converter provides PFR inertial support power, the frequency response characteristics of power system are shown as follows

$$
2 H \frac{\mathrm{d} \omega}{\mathrm{d} t}=P-P_{\mathrm{g}}=\left[P_{0}+P_{\mathrm{FR}}\right]-\left\{\left[P_{\mathrm{g} 0}+\Delta P_{g}(t)\right]-K_{g}\left(\omega_{\mathrm{N}}-\omega\right)\right\}=P_{\mathrm{FR}}+K_{\mathrm{g}}\left(\omega_{\mathrm{N}}-\omega\right)-\Delta P_{\mathrm{g}}(t)
$$

Substituting the expression of power disturbance shown in (1) and the expression of inertial support power shown in (2) into (3), the differential equation of angular frequency $\omega$ shown in the following formula is obtained.

$$
2 H \frac{\mathrm{d} \omega}{\mathrm{d} t}=-K_{\mathrm{d}} \frac{\mathrm{d} \omega}{\mathrm{d} t}+K_{\mathrm{g}}\left(\omega_{\mathrm{N}}-\omega\right)-\Delta P_{g}\left(1-e^{-t / \tau}\right)
$$

By solving the above differential equation, the expression of the system frequency can be obtained.

$$
\omega=\left\{\begin{array}{lr}
\omega_{\mathrm{N}}-\frac{\Delta P_{\mathrm{g}}}{K_{\mathrm{g}}}+\frac{\Delta P_{\mathrm{g}}}{K_{\mathrm{g}}-\left(2 H+K_{\mathrm{d}}\right) / \tau} e^{-t / \tau}+\left(\frac{\Delta P_{\mathrm{g}}}{K_{\mathrm{g}}}-\frac{\Delta P_{\mathrm{g}}}{K_{\mathrm{g}}-\left(2 H+K_{\mathrm{d}}\right) / \tau}\right) e^{\frac{-K_{\mathrm{g}}}{2 H+K_{\mathrm{d}}} t}, & \tau \neq \frac{2 H+K_{\mathrm{d}}}{K_{\mathrm{g}}} \\
\omega_{\mathrm{N}}-\frac{\Delta P_{\mathrm{g}}}{K_{\mathrm{g}}}+\left(\frac{\Delta P_{\mathrm{g}} t}{2 H+K_{\mathrm{d}}}+\frac{\Delta P_{\mathrm{g}}}{K_{\mathrm{g}}}\right) e^{\frac{-K_{\mathrm{g}}}{2 H+K_{\mathrm{d}}} t}, & \tau=\frac{2 H+K_{\mathrm{d}}}{K_{\mathrm{g}}}
\end{array}\right.
$$

It can be seen from the above formula that the time domain response of frequency includes steady-state component and exponential transient component. Among them, the steady-state component is a direct flow, which is determined by the strength of the disturbance power and the damping coefficient of the power grid itself. The greater the disturbance power intensity is, the weaker the grid damping is, and the more the DC component deviates from the rated frequency. Only when the intensity of disturbance power is zero, the DC component value is equal to the rated frequency.

\section{Simulation results of inertia response characteristics control technology for high voltage direct hanging energy storage system}

The simulation results show that when photovoltaic and wind power are connected to the grid at rated power $(50 \mathrm{MW})$ in $0 \mathrm{~s}$, the output power decreases to $20 \mathrm{MW}$ due to the fluctuation of illumination and wind speed in $30 \mathrm{~s}$. When the photovoltaic and wind power return to the rated value in $50 \mathrm{~s}$, the inverter is locked and the photovoltaic and wind power are off grid in $90 \mathrm{~s}$.

Fig. 5 shows the simulation waveforms of PV and wind power grid connection point frequency and output power. Fig. 6 shows the frequency, voltage and active and reactive output waveforms of $220 \mathrm{kV}$ energy storage system. It can be seen from the simulation waveform that when the output power of photovoltaic and wind power fluctuates, the system frequency will also change. The active power and reactive power of the system will be 
stabilized according to the system voltage and frequency.
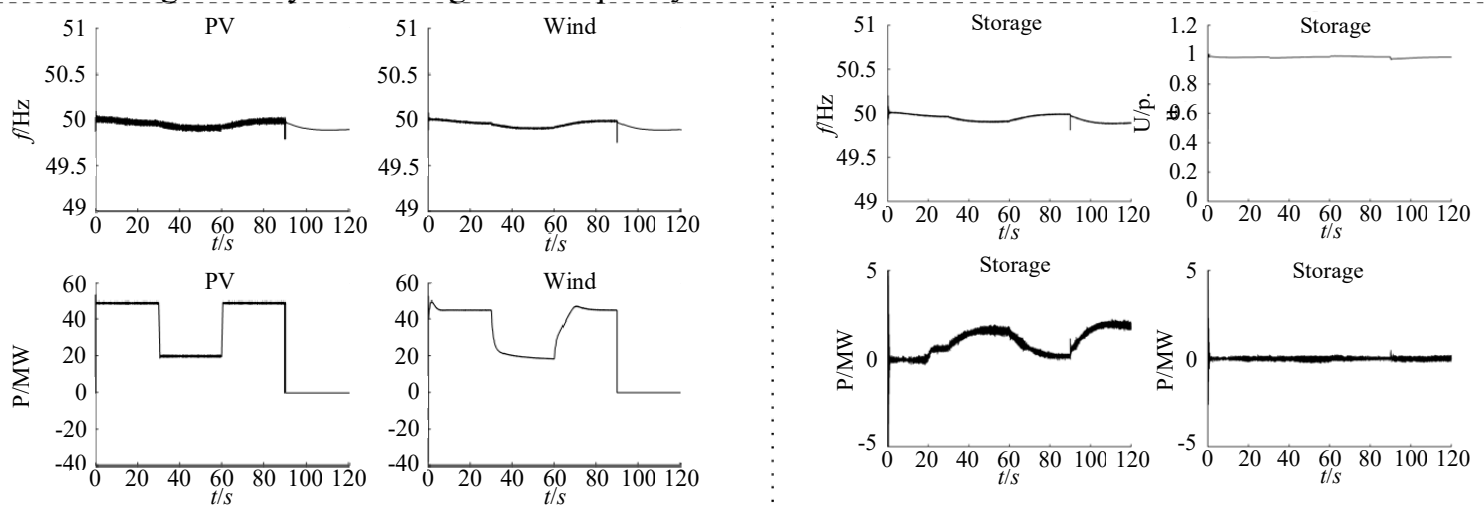

Figure 5. Frequency and output power waveform of PV and wind power grid.

Figure 6. Frequency, voltage, active and reactive output waveforms.

The simulation of load switching (50MW in 20s and $50 \mathrm{MW}$ in 25s) and generator off grid (in 30s, hydropower unit is off grid, 660MW load is cut off in 30.014s). Fig. 7 shows the voltage and frequency response waveforms of the system when the load is switched and the generator is off grid.

From the above simulation results, it can be seen that load switching and generator off grid will cause system


Figure 7. The voltage and frequency waveforms of load switching and generator off grid system are carried out.

\section{Conclusion}

This paper has studied inertial response control technology of high voltage direct hanging energy storage system. It is concluded that the intervention of energy storage system is helpful to flexibly control the inertia level and damping capacity of power system, and effectively restrain the weakening of frequency and voltage stability of power system caused by natural fluctuation and randomness of new energy.

\section{Acknowledgment}

This work was supported by Science and Technology Project of State Grid Jiangsu Electric Power Co., Ltd, i.e., frequency fluctuation and $220 \mathrm{kV}$ bus voltage fluctuation. There is power and reactive power transmission, and the transmission power presents low-frequency oscillation, with the oscillation frequency of about $1.8 \mathrm{~Hz}$. It is seen from Fig. 8 that the energy storage system can realize the stable operation of voltage and frequency through the coordinated control of inertia and damping.

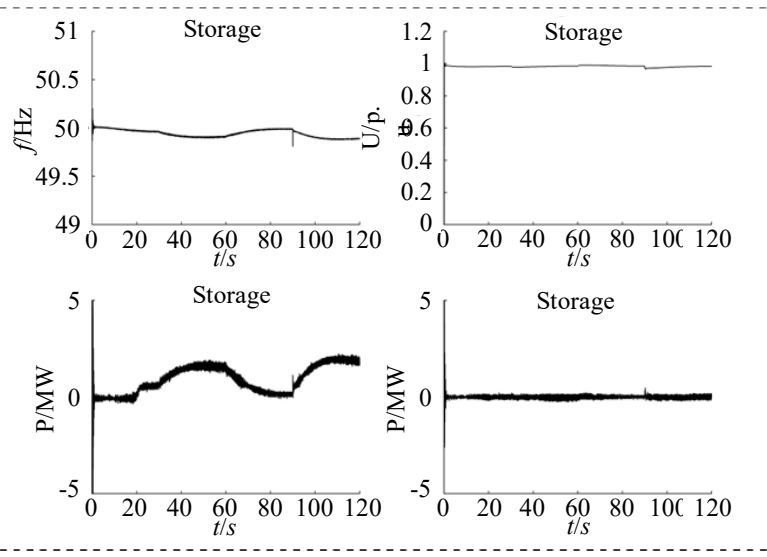

Figure 8. System voltage and frequency waveform when energy storage adopts coordinated control of inertia and damping off grid system are carried out.
Research on optimization control technology for efficient utilization of medium voltage direct hanging energy storage system supporting the construction of shared energy storage power station (J2020013).

\section{References}

1. Mei N., Yuan B., Li T., et al. (2018) Study on control strategy of bipolarise station connected to islanded renewable power plant [J]. Power System Technology, 42: 3575-3582.

2. Z. Xiaolin, D. Wei, Y. Guanzxiu and Z. Junjun. Test method for inertia and damping of photovoltaic virtual synchronous generator based on power angle transfer function [J]. 2018 2nd IEEE Conference on Energy Internet and Energy System Integration (EI2), 
Beijing, 2018, pp. 1-5.

3. C. Root, H. Presume, D. Proudfoot, L. Willis and R. Masiello. Using battery energy storage to reduce renewable resource curtailment [J]. 2017 IEEE Power \& Energy Society Innovative Smart Grid Technologies Conference (ISGT), Washington, DC, 2017, pp. 1-5.

4. M. Qian, D. Zhao, J. Ma, D. Jiang, M. Ding and L. Xiang. A Centralized frequency regulation strategy of PV power plant based equal adjustable capacity proportion mode $[\mathrm{J}] .2018$ China International Conference on Electricity Distribution (CICED), Tianjin, 2018, pp. 1797-1801.

5. Li G.J., Li H.Y., Wu F.B and Yin S. (2020) Equalization control strategy of medium voltage direct hanging energy storage system [C]. 2020 International Conference on Energy, Environment and Bioengineering, ICEEB 2020, 185: 01085. 